Current recommended doses range from $0 \cdot 10$ $1.48 \mu \mathrm{mol} / \mathrm{kg} /$ day, ${ }^{45}$ and some recommend that copper be excluded completely from short term parenteral nutrition. ${ }^{6}$ Our experience suggests that potentially toxic concentrations of copper can be achieved in critically ill preterm infants and that intravenous copper supplements should be withheld until the babies are stable, and then their serum copper concentrations should be monitored. Further studies are needed to define optimal intravenous copper requirements.

We are grateful to Dr RPH Thompson, Dr B Slavin, and Professor JW Scopes for their support.

\footnotetext{
References

1 Al-Rashid AA, Spangler J. Neonatal copper deficiency. N Engl J Med 1971;285:841-3.
}

${ }^{2}$ Askenazi A, Lewis S, Djaldetti M, Fishel M, Benvenish D. The syndrome of neonatal copper deficiency. Pediatrics 1973;52:525-32.

${ }^{3}$ Heller RM, Kirchner SG, O'Neill JA, et al. Skeletal changes of copper deficiency in infants receiving prolonged total, parenteral nutrition. J Pediatr 1978;92:947-59.

${ }^{4}$ Sutton AM, Harvie A, Cockburn F, Farquharson J, Logan RW. Copper deficiency in the preterm infant of very low birthweight. Arch Dis Child 1985;60:644-51.

5 James BE, Hendry PG, MacMahon RA. Total parenteral nutrition of premature infants. 2. Requirements for micronutrient elements. Aust $J$ Paediatr 1979;15:67-71.

6 Casey CB, Hambidge KM. Trace minerals. In: Tsang RC, ed. Vitamin and mineral requirements in preterm infants. New York: Marcel Dekker, 1985;153-84.

Correspondence to Dr Karen Simmer, Department of Paediatrics, Flinders Medical Centre, Bedford Park, South Australia 5042.

Received 13 July 1987

\title{
Maternal narcotic abuse and the newborn
}

\author{
L G ALROOMI, ${ }^{*} \mathrm{~J}$ DAVIDSON, $\dagger \mathrm{T} \mathrm{J}$ EVANS, $\uparrow$ P GALEA, ${ }^{*}$ AND R HOWAT* \\ ${ }^{*}$ Glasgow Royal Maternity Hospital and †Stobhill General Hospital, Glasgow
}

SUMMARY In 50 infants born to women who continued to take heroin during all or part of their pregnancy the drug withdrawal symptoms were mild and were noted in 21 infants $(42 \%)$. Only nine infants required treatment. Sudden infant death syndrome occurred in two infants at 4 and 6 months.

An increasing number of infants are being born to mothers addicted to narcotic drugs. Reports about the effects of maternal drug abuse on the infant are conflicting. Previous series, largely from North America, have reported increased neonatal mortality and morbidity in these infants. ${ }^{12}$ Recently, Klenka reported minor withdrawal symptoms in more than $70 \%$ of infants but noted a low incidence of severe symptoms. ${ }^{3}$ This was attributed to smoking heroin rather than to intravenous abuse. In order to ascertain the current risk we studied 50 infants born between 1983 and 1986.

\section{Patients and methods}

During the study period, 44 women known to be addicted to narcotic drugs delivered 50 infants in two Glasgow maternity hospitals. One mother had a twin pregnancy, and five women delivered twice during this period. The combined annual delivery rate in these two hospitals is roughly 6000 .

All except one of the mothers were using heroin, either alone or in combination with a variety of other drugs. The one exception was a methadone addict. The mean (SD) duration of addiction before delivery was $2.7(1.5)$ years. The amount of heroin used varied widely and was difficult to ascertain. Most of the women (75\%) were intravenous heroin abusers; the rest either smoked or snorted the drug. Twenty four women had partners who were also drug addicts.

The ages of the mothers ranged from 17-34 years: mean (SD) 21.5 (3.4). All were offered routine antenatal care with the addition of an inpatient detoxification programme. Nineteen of the women had little or no antenatal care, and two concealed their pregnancies.

The infants were admitted routinely to the special care nursery after delivery and were observed for signs of drug withdrawal for 48 hours. Symptomatic infants remained under close observation. Treatment was initiated if there were constant tremulous movements, convulsions, or a disturbed sleep or behavioural pattern such as restlessness or crying within one hour of a feed. The drugs used in treatment were phenobarbitone, chloral hydrate, and phenobarbitone either singly or in combination depending on the individual clinician. 


\section{Results}

Signs of drug withdrawal were noted in 21 infants $(42 \%)$ (table 1). In most of the infants these were mild but nine $(18 \%)$ had symptoms considered sufficient to warrent treatment. Five had late or prolonged withdrawal symptoms with irritability and excessive hunger.

The birth weight of affected infants ranged from $1.1-3.54 \mathrm{~kg}$ : mean (SD) $2.77(0.58)$. Neonatal problems, other than drug withdrawal symptoms, in the infants of addicted mothers are documented in table 2 . In 19 pregnancies there had been little or no antenatal care and $11(57 \%)$ of them resulted in preterm delivery. Thirty nine $(78 \%)$ of the infants were discharged home, eight under supervision orders. Nine were discharged to foster care, and two were adopted.

Few of these children were followed up successfully. Most failed to attend outpatient appointments. Three are known to have long term problems. One child is microcephalic and severely handicapped, one has a cleft palate, and one is deaf. There were no neonatal deaths in this group. Two infants suffered sudden infant death syndrome at 4 and 6 months.

Table 1 Drug withdrawal symptoms in 21 affected infants

\begin{tabular}{ll}
\hline Symptom & No of infants $(\%)$ \\
\hline Jitteriness & $21(100 \cdot 0)$ \\
High pitched cry & $11(52 \cdot 4)$ \\
Snuffles & $10(47 \cdot 6)$ \\
Sleep disturbance & $9(42 \cdot 9)$ \\
Vomiting & $6(28 \cdot 6)$ \\
Abnormal feeding pattern & $5(23 \cdot 8)$ \\
Tachypnoea & $4(19 \cdot 0)$ \\
Fever/excess sweating & $1(4 \cdot 8)$ \\
Convulsions & $1(4 \cdot 8)$ \\
\hline
\end{tabular}

Table 2 Neonatal complications occurring in infants $(n=50)$ of addicted mothers

\begin{tabular}{ll}
\hline Complication & No of infants (\%) \\
\hline Preterm delivery (<37 weeks) & $14(28)$ \\
Small for gestational age & $14(28)$ \\
$\begin{array}{l}\text { Occipitofrontal head circumference } \\
\quad<10 t h \text { centile }\end{array}$ & $17(34)$ \\
$\begin{array}{l}\text { Delivery by caesarean section } \\
\text { Delivery by forceps }\end{array}$ & $7(16)$ \\
$\begin{array}{l}\text { Fetal distress } \\
\text { (cardiotocographic changes with or }\end{array}$ & $18(36)$ \\
$\quad$ without meconium staining) & $14(28)$ \\
$\begin{array}{l}\text { Apgar scores <6 at one minute } \\
\text { Idiopathic respiratory distress }\end{array}$ & $4(8)$ \\
$\quad$ syndrome & $3(6)$ \\
\hline
\end{tabular}

\section{Discussion}

The incidence and severity of drug withdrawal symptoms in the group of infants studied is lower than previously reported in infants of narcotic addicts. Klenka attributed a reduced severity of symptoms to low dose exposure in mothers who smoked rather than injected heroin. ${ }^{3}$ In our series, however, $75 \%$ were intravenous drug abusers.

Alterations in the severity of symptoms may reflect changing patterns of drug taking: most addicts now take more than one drug. The infant may be affected not only by exposure to heroin but by the other drugs taken. It is well recognised that methadone results in more prolonged withdrawal symptoms. Most mothers in our series were using heroin in combination with other drugs but few were using methadone on a regular basis.

No neonatal deaths occurred in our series. There were two cases of sudden infant death syndrome; an increased incidence has been previously reported in infants of drug addicts. ${ }^{4}$ In keeping with previous reports, we noted suboptimal intrauterine growth, including head growth, in many infants. ${ }^{5}$ This may relate as much to other factors in the lifestyle of the addict as to intrauterine heroin exposure. Smoking, poor diet, and poor antenatal care, all associated with poor fetal growth, are common in these mothers. Where there was little or no antenatal care we noted an increased risk of preterm delivery. This is well recognised in non-addict populations. Perhaps efforts to improve the outlook for these infants should be directed at encouraging the use of antenatal care resources.

The ability of the drug addict to care for her child after discharge is of major concern. Many addicts have unstable backgrounds with no family support. An early assessment of the family and support available to the mother is essential. Debate continues about the long term outlook for these children; there are reports of an increased incidence of behaviour problems and learning difficulties. ${ }^{6}$ Due to difficulties with follow up we were unable to explore this.

No mother in our group was positive for human immunodeficiency virus. With the increasing prevalence of this virus this may well become one of the major risks to these infants in the future.

In conclusion we report a low incidence of severe drug withdrawal symptoms requiring treatment in infants born to drug addicted mothers. The main risks were associated with the mother's lifestyle as a drug addict rather than a direct effect of the drugs.

We thank our obstetric and paediatric colleagues for allowing us to report on their patients and Miss A Campbell for typing the manuscript. 
References

1 Zelson C, Rubid E, Wassermann E. Neonatal narcotic addiction: 10 year observation. Pediatrics 1971;48:178-89.

2 Fricker HS, Segal S. Narcotic addiction, pregnancy and the newborn. Am J Dis Child 1978;132:360-6.

3 Klenka HM. Babies born in a district general hospital to mothers taking heroin. $\mathrm{Br}$ Med $J$ 1986;293:745-6.

4 Chavez CJ, Ostrea EM, Stryker JC. Sudden infant death syndrome among infants of drug dependent mothers. $J$ Pediatr 1979;95:407-9.
5 Lifschitz MH, Wilson GS, O'Brien Smith E, Desmond MM. Fetal and postnatal growth of children born to narcoticdependent women. J Pediatr 1983;102:686-91.

6 Wilson GS, McCreavy R, Kean J, Baxter JC. The development of preschool children of heroin-addicted mothers: a controlled study. Pediatrics 1979;63:135-41.

Correspondence to Dr L AlRoomi, Paediatric Department, Glasgow Royal Maternity Hospital, Rottenrow, Glasgow G4 0NA.

Received 12 August 1987

\title{
Neonatal infections with Haemophilus species
}

\author{
L M MILNE, ${ }^{*}$ D ISAACS, $\dagger$ AND P J CROOK* \\ ${ }^{*}$ Public Health Laboratory and †Department of Paediatrics, John Radcliffe Hospital, Oxford
}

SUMMARY During a 27 month study seven nonserotypable strains of Haemophilus influenzae and two of Haemophilus parainfluenzae were isolated from nine neonates. Seven had early infection associated with respiratory distress or conjunctivitis; three had septicaemia one of whom died. The incidence of haemophilus septicaemia was 0.23 per 1000 live births.

The number of reports of infections caused by nonserotypable Haemophilus influenzae during the neonatal period is increasing. ${ }^{1}$ Any series that includes only cases of bacteraemia or meningitis is likely to under report characteristic clinical features of infection with this organism. ${ }^{2}$ We report a study conducted over 27 months of all haemophilus isolates in one unit.

\section{Material and methods}

On 1 January 1985 surveillance of haemophilus infections in our neonatal unit started and we report the results until 31 March 1987. When systemic sepsis was suspected we took samples of blood, urine, cerebrospinal fluid, nose, throat, umbilical, and rectal swabs before starting antibiotics. In addition, endotracheal secretions from babies requiring assisted ventilation were routinely cultured three times a week.

Blood cultures were performed using brain heart infusion broth with and without sodium thioglycollate (Gibco Ltd) with routine subculture to chocolate agar after 48 hours and five days' incubation at $37^{\circ} \mathrm{C}$. Haemophilus spp were initially identified by their growth and morphology on chocolate agar after primary culture or following enrichment in cooked meat medium. Strains were speciated and biotyped according to the method of Kilian using laboratory prepared media. ${ }^{3}$ Slide agglutination was used to attempt to serotype the strains using type specific antisera prepared by the Public Health Laboratory Service (type b) and Wellcome (types a, $c$, d, e, and f).

Antibiotic sensitivities were performed and interpreted using the comparative method. ${ }^{4}$ To prepare the inoculum six isolated colonies were touched and added to $2.5 \mathrm{ml}$ of $1 \%$ tryptone water (Oxoid). $H$ influenzae NCTC 11931 was used as the control organism. The test medium was Isosensitest (Oxoid) with 5\% saponin lysed horse blood (Tissue Culture Services) and $10 \mathrm{mg} / \mathrm{ml} \beta$ nicotinamide adenine dinucleotide grade III (Sigma). Incubation was performed at $37^{\circ} \mathrm{C}$ in a $5 \%$ carbon dioxide incubator. Disc contents (measured in $\mu \mathrm{g}$ ) were ampicillin 2 , cefuroxime 30 , gentamicin 10 , netilmicin 30 , neomycin 10 , trimethoprim $1 \cdot 25$, chloramphenicol 10 (Oxoid) and polymyxin B 250 units (Mast Laboratories Limited). The presence of $\beta$ lactamase was detected with Intralactam strips (Mast Laboratories Limited).

\section{Results}

Throughout the study there were 12910 live births in the hospital. The characteristics of the nine babies with Haemophilus are shown in the table. All neonates were born by spontaneous vertex delivery, except cases 1 and 9 who were delivered by caesarean section. Cases 1 and 2 grew $H$ influenzae and $H$ parainfluenzae, respectively, from respiratory secretions at 3 weeks of age. These were probably nosocomial infections as the two babies were not 